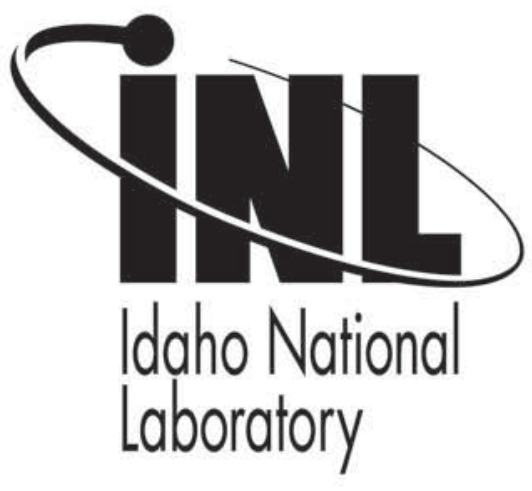

\title{
Modeling and Simulating Blast Effects on Electric Substations
}

\section{IEEE International Conference on Technologies for Homeland Security}

Lyle G. Roybal

Robert F. Jeffers

Kent E. McGillivary

Tony D. Paul

Ryan Jacobson

May 2009

This is a preprint of a paper intended for publication in a journal or proceedings. Since changes may be made before publication, this preprint should not be cited or reproduced without permission of the author. This document was prepared as an account of work sponsored by an agency of the United States Government. Neither the United States Government nor any agency thereof, or any of their employees, makes any warranty, expressed or implied, or assumes any legal liability or responsibility for any third party's use, or the results of such use, of any information, apparatus, product or process disclosed in this report, or represents that its use by such third party would not infringe privately owned rights. The views expressed in this paper are not necessarily those of the United States Government or the sponsoring agency. 


\title{
Modeling and Simulating Blast Effects on Electric Substations
}

\author{
Lyle G. Roybal ${ }^{1}$, Robert F. Jeffers ${ }^{1}$, Kent E. McGillivary ${ }^{1}$, Tony D. Paul ${ }^{1}$, Ryan Jacobson ${ }^{2}$ \\ ${ }^{1}$ Idaho National Laboratory, 2525 North Fremont Avenue \\ Idaho Falls, Idaho, 83415 \\ [lyle.roybal, robert.jeffers, kent.mcgillivary, tony.paul]@inl.gov \\ ${ }^{2}$ University of Wyoming, 1000 E. University Avenue \\ Laramie, WY 82071
}

\begin{abstract}
A software simulation tool was developed at Idaho National Laboratory to estimate the fragility of electric substation components subject to an explosive blast. Damage caused by explosively driven fragments on a generic electric substation was estimated by using a ray-tracing technique to track and tabulate fragment impacts and penetrations of substation components. This technique is based on methods used for assessing vulnerability of military aircraft and ground vehicles to explosive blasts. An open-source rendering and ray-trace engine was used for geometric modeling and interactions between fragments and substation components. Semiempirical material interactions models were used to calculate blast parameters and simulate high-velocity material interactions between explosively driven fragments and substation components. Finally, a Monte Carlo simulation was added to model the random nature of fragment generation allowing a skilled analyst to predict failure probabilities of substation components.
\end{abstract}

\section{INTRODUCTION}

Critical infrastructure protection against both natural hazard and terrorist initiated events has been a significant area of concern since the events of September 11, 2001 prompting creation of the Department of Homeland Security in the United States. Significant effort and resources have been devoted to modeling and predicting the behavior of infrastructure systems, such as the electric power grid, communications networks, and natural gas distribution systems (for example), during and after disaster scenarios whether from natural causes accidental in nature or initiated by terrorist activity. The fidelity of the infrastructure models available varies from very detailed physics-based models capable of predicting transient behavior of individual infrastructure components, to low fidelity models that concentrate more on the general steady-state behavior of infrastructure systems and the socio-economic impact of infrastructure failure. Detailed physics-based models tend to be deterministic in nature, while more generalized models are more suitable for parametric studies. Both approaches rely on the use of fragility models to predict the failure of basic components as a function of the hazard level. Fragility functions are used in the infrastructure models at their lowest levels, and provide the forcing functions that ultimately determine how the rest of the model responds.

The Critical Infrastructure and Resilience Simulator $\left(\mathrm{CIPR}^{\mathrm{sim}}\right)$ simulation environment developed by Idaho National Laboratory (INL) for the Office of the Secretary of Defense (OSD) US Strategic Command (STRATCOM) has taken some important first steps in modeling and predicting the behavior of infrastructures. However, the development and implementation of the CIPR $^{\text {sim }}$ has exposed important and serious data gaps with respect to modeling the failure of basic electric power substation components, such as transformers, switches, porcelain insulators, cell tower structures, cell tower supporting electronics, gas compressor stations, etc. The list is nearly endless. Failure data for these types of components are very sparse for natural hazards, such as earthquakes and hurricanes. But, for explosive events, there are no data available that address component failure at any level. A simulation tool developed by INL fills that data gap with credible analysis that is based on proven methodologies used in the aerospace and military arenas. These methodologies have previously been used to calculate component failure probability of space assets, aircraft, and ground vehicles to exploding and incendiary devices. Moreover, the methods presented herein are designed to model the stochastic nature of initiated and natural hazard events, and provide a natural feed into Monte Carlo methods for modeling infrastructure response in a parametric fashion. 


\section{APPROACH}

The goal of this work was to develop a methodology and ultimately a software simulation tool to estimate the fragility of electric substation components to explosive blasts. The approach used was based on similar methods used by the defense and military modeling and simulation communities for estimating the fragility of aircraft and ground vehicles to proximity surface-to-air-missiles (SAMS). The defense industry has developed and used methodologies for evaluating the vulnerability of military aircraft and ground vehicles to missile, mortar, and landmine-type explosive devices using geometric models of assets combined with simple materials interaction models. Here, fragments from military warheads were modeled as they traveled through and interacted with an asset model. Using this analogy, fragments from a simulated explosion were tracked as they encountered various components in a geometric model of an electric substation. Material interactions between fragments and substation components were also modeled, including component penetration and estimated loss of fragment velocity and mass. Failures of individual substation components were tabulated using either penetration or impact on the component as a measure of failure.

This approach maximized existing software algorithms used by military research analysts and included validated methods and algorithms taken from the Conventional Weapons Effects Program (CONWEP) library, which is a software implementation of the methods described in the Design and Analysis of Hardened Structures to Conventional Weapons Effects [1] published jointly by multiple military branches. Also, the BRL-CAD [2] ray-tracer available as open source software was used to trace fragments through a geometric model of the substation. This BRL-CAD software was developed by the Ballistic Research Laboratory (now the Army Research Laboratory) specifically for aircraft and ground vehicle vulnerability analysis to conventional military weapons.

This technical approach essentially consists of four major areas: (1) modeling the explosion, (2) modeling the geometry of the explosion and critical infrastructure asset, (3) modeling the interaction of the explosively driven fragments and infrastructure components, and (4) tabulating the effects of the interactions between fragments and components to estimate component fragility. Because of the random nature of the fragment generation with respect to fragment size and initial direction, the simulation included a Monte Carlo wrapper around the core software model to calculate the probabilities of component damage or destruction for a given explosion size/type and location with respect to the electric substation assets. Short descriptions of each of these steps follow.

\section{BLAST SIMULATION}

First, a simple model of an Improvised Explosive Device (IED) was developed that emphasized the generation and initial conditions of fragments created by the explosion. For this work, the effects of explosively driven fragments and their impact on substation components were of primary concern. The blast wave effects were of secondary concern, although it can be shown that the methods used can easily calculate blast wave loading on components using the presented area functions available from the BRL-CAD ray-trace code.

A representative IED designed primarily to generate large numbers of fragments was simulated by assuming distribution of fragment sizes about the body of the container holding the explosive propellant. Fragment velocities were calculated using the algorithms and libraries published in the "Design and Analysis of Hardened Structures to Conventional Weapons Effects" technical manual published by the Departments of the Army, Air Force, Navy, and Defense Special Weapons Agency [1]. Initial fragment exit speeds were analytically calculated using the Gurney equations for cylinder of Ammonium Nitrate - Fuel Oil (or ANFO) propellant. ANFO represents a common and easily manufactured explosive of choice for terrorists and terrorist organizations. The ANFO configuration assumed was an $87 \%$ Trinitrotoluene (TNT) equivalent blast. Because the fragments generated were considered small compared to the initial blast, all fragments have the same initial exit speed from the point of the explosion. Typical exit fragment speeds are $2000 \mathrm{~m} / \mathrm{s}$. Reduction of downrange ballistic fragment velocity varies considerably and is a function of fragment size, shape, and weight.

The final piece of the blast simulation task was to perform random draws on fragment mass and fragment direction. Fragment masses were drawn from six discrete possibilities ranging from 17 to 182 grams. Each mass category was assumed to have the same total mass (number of fragments times fragment mass). This meant that for a given explosion, there were more light fragments than heavy fragments.

\section{FRAGMENT/ASSET GEOMETRY}

The geometry of the interaction of the fragments with the electric substation and its components was done by building a three-dimensional constructive solid geometry model of a representative substation, and using a shot-line technique to trace each fragment as it moved in space away from the explosion site. The ray-tracing/shot-line analysis techniques were developed in the 1980's as a way to predict the damage to a target caused by ballistic impact of projectiles. The shot-line method involves projecting rays representative of a ballistic projectile's path through a target. The result is a sequential list of components or parts of a target encountered along the shot-line assuming a straight line path of the projectile. 
Initially, the military and its contractors developed two main simulation tools to do this analysis. The FASTGEN code was promoted mainly by the Navy for aircraft simulation, and the BRL-CAD code was developed by the Army's Ballistic Research Laboratory with primary emphasis on ground vehicle (tank) analysis. FASTGEN uses faceted triangular plates to form surfaces of the target of interest, while BRL-CAD uses constructive solid geometry (CSG) techniques to form the asset model. Both techniques have been used in the animation industry for rendering computer graphics images.

For this effort, the BRL-CAD system was chosen for generating shot-lines of the electric substation model. This was done because CSG techniques are better suited for modeling internal components of assets, and the BRL-CAD software system is available as an open-source resource. This allowed for the modification of the ray-trace software as needed to suit specific analysis requirements.

In itself, BRL-CAD is a powerful solid modeling system designed to interactively create and analyze three-dimensional geometric models. It includes an interactive geometry editor for building targets complete with error checking and display modules. Constructive solid geometry techniques allow a modeler to create a complex surface or object by using Boolean operators to combine objects. Often, CSG presents a model or surface that appears visually complex, but is actually little more than cleverly combined basic geometric objects. The simplest solid objects used for the representation are called primitives. Typically, they are the objects of simple shape: cuboids, cylinders, prisms, pyramids, spheres, and cones (for example). Objects are constructed from primitives by means of allowable Boolean operations. The BRL-CAD ray-tracer used unions, intersections, and differences. Examples of simple CSG operations can be illustrated by using a block and a sphere, and combining them with union, difference, and intersection operations. The results are shown in Figure 1.
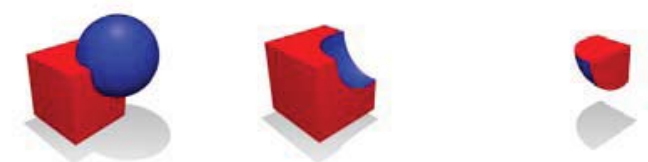

Figure 1 - Example CSG operations [3].

Very complex entities can be constructed by using these three simple operators combined with box, sphere, and cylinder shapes as illustrated in Figure 2.

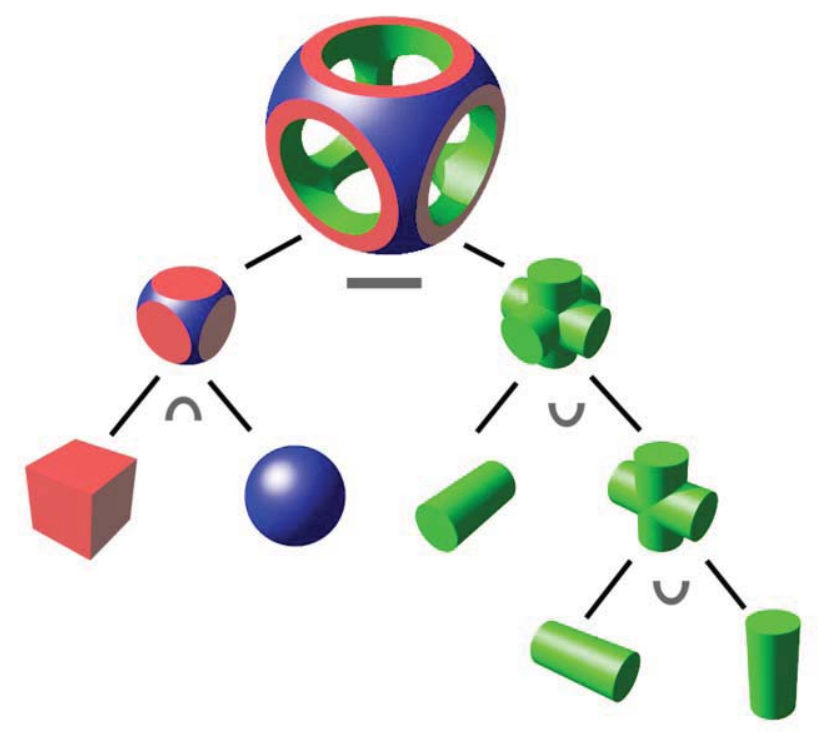

Figure 2 - Example complex CSG shape [3].

A complete CSG model of a representative electric substation was built using the BRL-CAD interactive modeler. This model consisted of over 500 individual components, such as circuit breakers, transformers, and lightning arrestors. The BRL-CAD model system can assign material attributes for each component. These attributes include material type (aluminum, glass, copper, etc.), and surface properties, such as reflectivity. Surface property information is important for graphics rendering. However, material type, thickness, and incident angle are the most important data extracted by the ray-tracer from the line-of-sight shot-line data, and are later used by materials interactions models. An example breaker assembly modeled using this approach is shown below. Figure 3 shows an actual photograph of a breaker assembly. Figure 4 shows the CSG representation used for the shot-line analysis. The breaker assembly modeled in Figure 4 consists of steel tanks, aluminum shields, glass insulators, and copper conductors, as well as a steel C-channel understructure.

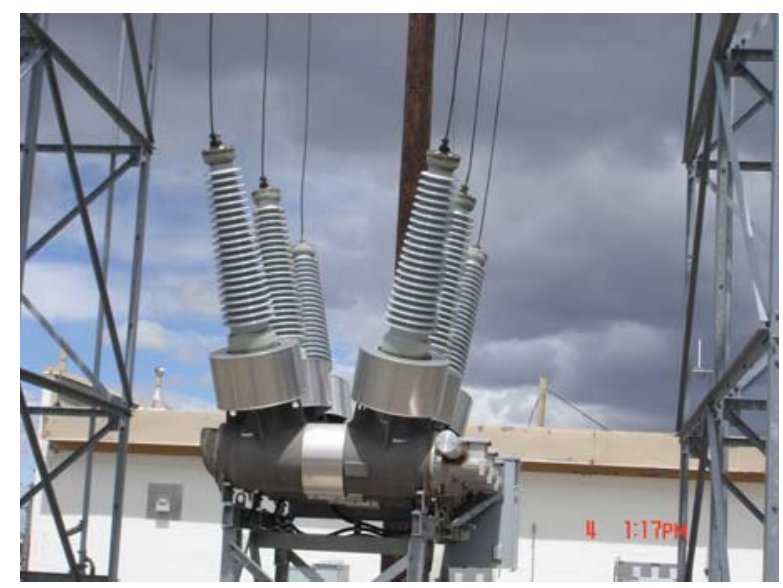

Figure 3 - Breaker Assembly Photograph. 


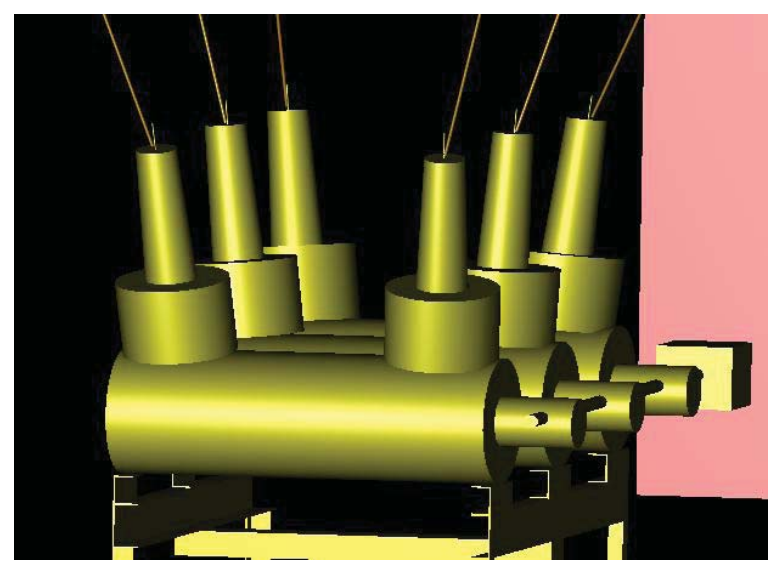

Figure 4 - Breaker Assembly CSG Model.

Once a comprehensive model of the substation was completed, shot-line data from each fragment generated by the simulated explosion were tabulated by the BRL-CAD ray-trace engine. Information provided by the ray-tracer can be tailored to suit the analysis needs. In this case, INL personnel modified the ray-trace software to provide tabulated output that included fragment properties as well as the impacted component properties. Tabulated fragment properties include the fragment mass and its initial velocity (identical for all fragments), and distance traveled from the origin of the explosion. The tabulated properties for each component encountered included component name, material type, angle of incidence, apparent thickness of material, and exit angle of the fragment. This was done for every component encountered along the fragments' travel path. The tabular data from a single fragment is often called the ray-stack data. These data can be of variable length depending on the number of components and primitives encountered by a given fragment. Initially, the ray-stack data are compiled by the ray-trace software without regard to whether the fragment actually penetrates the components encountered. Graphically, shot-line data are extracted as shown in Figure 5. Here, a fragment is passed through critical components without regard to the material interaction process and is strictly geometrical in nature.

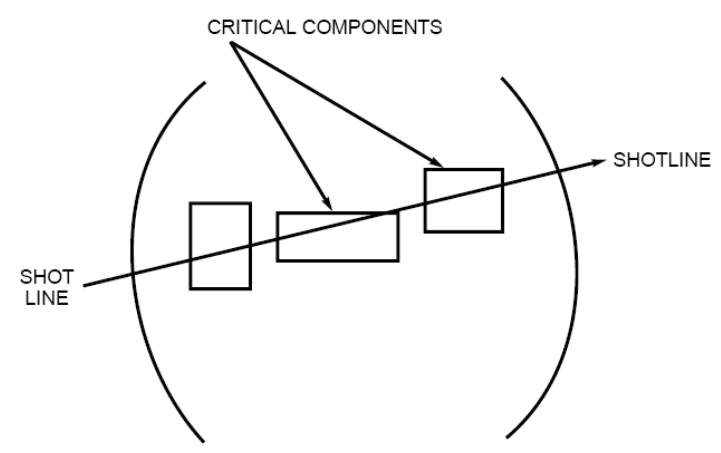

Figure 5 - Fragment Shot-line.
Once the ray-stack data are derived, each shot-line is analyzed individually for penetration and damage to components encountered. This starts with an evaluation of the initial impact velocity of the fragment onto the first component encountered along the shot-line. The striking velocity for the first component encountered by a given fragment can be calculated using equations 1 and 2 [1].

$$
\begin{aligned}
& V_{s}=V_{o} e^{-\left(a_{d} R / m_{f}^{0.333}\right)} \\
& a_{d}=(0.5)\left(C_{d}\right)(\rho) / k^{0.667}
\end{aligned}
$$

Where: $V_{\mathrm{s}}=$ striking velocity

$\mathrm{V}_{\mathrm{o}}=$ initial velocity

$\mathrm{R}=$ distance from detonation

$\mathrm{m}_{\mathrm{f}}=$ fragment mass

$\mathrm{C}_{\mathrm{d}}=$ drag coefficient

$\rho=$ air density

$\mathrm{k}=$ fragment shape factor

The initial impact velocity is affected by the initial velocity, the distance traveled by the fragment to first impact, the fragment mass, and a fragment shape factor. For this study, the fragments were considered nearly spherical. The component is evaluated for penetration by the fragment once the impact velocity of the fragment is calculated. A series of semi-empirical equations based on data and theory presented in reference 1 are used to calculate penetration depth into metals and concrete. If penetration was predicted, then a second series of equations were utilized to predict the mass and the velocity loss of the fragment given penetration. The calculated retained mass and velocity of the fragment were then used as initial conditions for the next component or surface encountered along the ray-stack. This process was repeated until the fragment was either stopped by the encountered component, or it passed through the entire ray-stack which means that every component along the fragment shot-line was penetrated.

Material interaction models and data exist for aluminum, steel, copper, glass, concrete, and other materials not generally found in industrial electric substations [1]. Additionally, models were added to simulate interaction with transformer oil. Equations 1 and 2 were adapted to calculate the loss of velocity given that a fragment travelled though a fixed distance in oil. In this case, the coefficient of drag and air density parameters were replaced with values suitable for oil.

Each fragment was traced through the substation geometric model by the BRL-CAD software module. This resulted in a table of penetrated or failed components that could be directly attributed to impact from blast fragments propelled by an explosion. 


\section{MONTE CARLO AND BOOKKEEPING}

The process described above provides an estimate of the damage caused by explosively propelled fragments for a single assumed instance and orientation of an explosion. In addition to the geometric modeling effort, bookkeeping algorithms were also added to account for fragment impacts and penetrations indicated on the material interactions models. The code was surrounded by a software wrapper that incorporated a Monte Carlo simulation loop around the entire simulation from fragment generation to impact results. For each Monte Carlo loop, the bomb orientation was perturbed to introduce randomness into the fragment flights, which influenced the probability of components being hit by the fragments. This analysis corresponded to a single bomb location consisting of 10,000 Monte Carlo simulations where the fragment flights were disturbed. The probabilities of components being impacted and penetrated were tabulated for each simulation. For each loop in the simulation, the size of the fragment was drawn from a discrete selection of six different fragment sizes with fixed numbers of each fragment size. The run time for a Monte Carlo simulation of 10,000 instances was less than five minutes on a workstation-class personal computer.

\section{Results AND Conclusions}

Much of the initial effort was dedicated to building a usable model of an electric substation. A useable model is defined as a model that contains sufficient fidelity to address any shielding concerns from structural components of the electric substation, such as I-Beams, C-channels, buildings, and concrete footings. Also, the model must provide some detail of the internal makeup of major components. For example, the main transformers consist of steel box tanks filled with oil with an internal copper core representing the windings of the transformer. This way, realistic penetration calculations through the outer skin, damped by oil, and impinging on the copper core could be made. Figure 6 shows a rendered model of a medium-sized substation that was used in the simulation. Figure 7 shows a photograph of the substation that the model in Figure 6 was based on.

The large green components in Figure 6 are the high-voltage transformers for the substation. Although difficult to see in the figure, they are complete with cooling fins, control boxes, insulators, tank structure, and core. The three large yellow objects on the left side of the model are lightning arrestors with support hardware, such as I-beams and C-channels. The large blue-green object towards the back of the model is the control building. Internally, this building consists of secondary voltage breakers and switch cabinets, and other control hardware needed by the substation. The three tall tower structures hold the high-voltage horn-gap switches for all three phases entering and leaving the substation. The breaker assembly shown in Figure 5 is located on the ground between towers two and three. Other prominent pieces of equipment include the high-voltage switchgear on the shorter towers and the smaller grounding transformers.

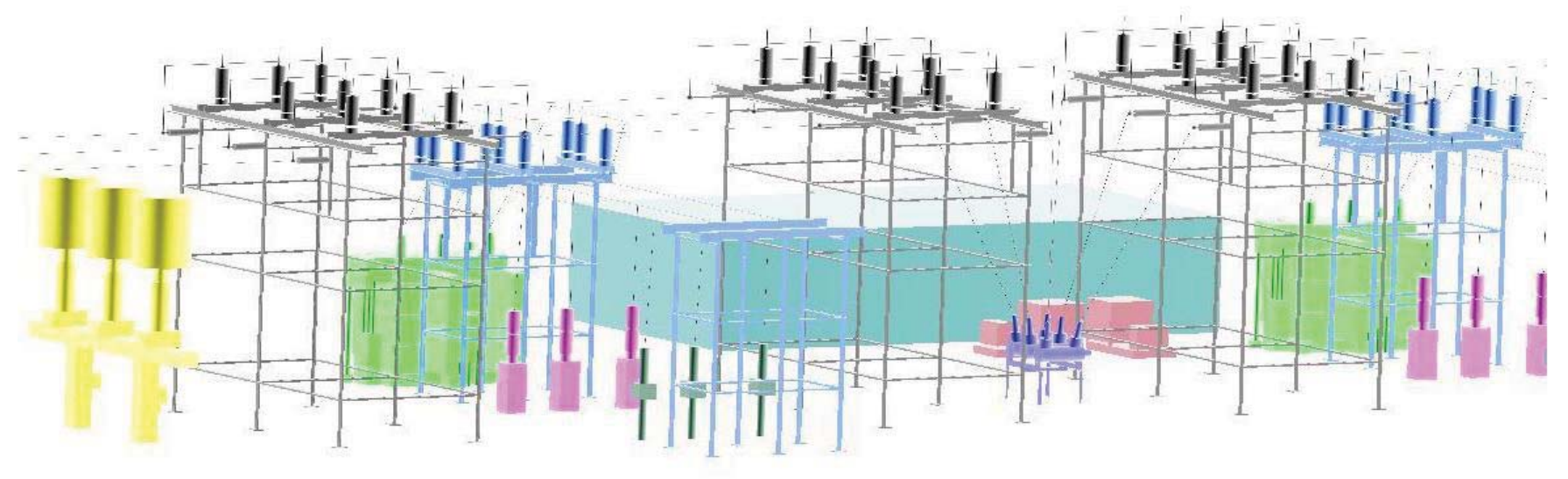

Figure 6 - Rendered graphic of the electric substation model used. 


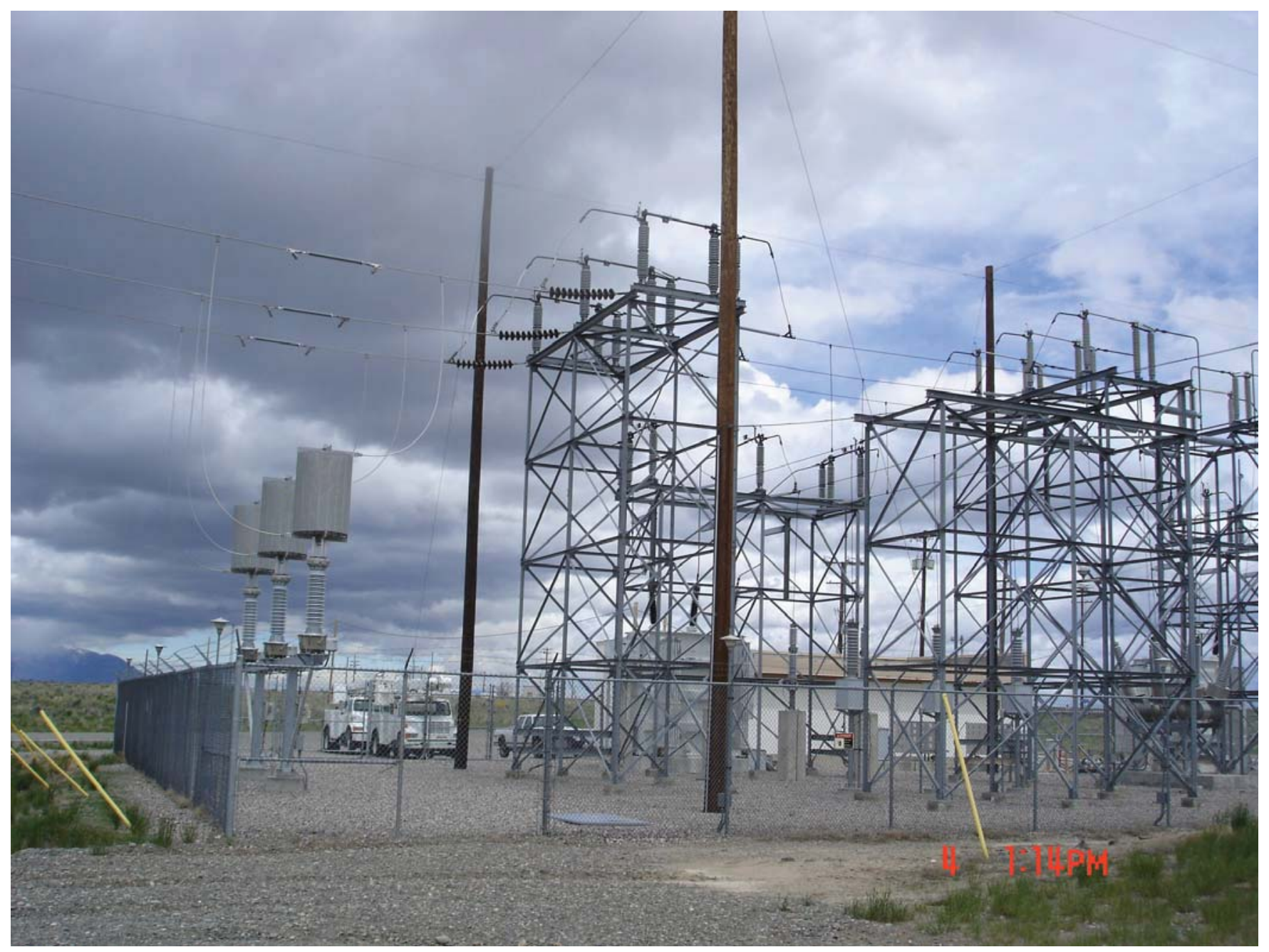

Figure 7 - Photograph of an electric substation which the CSG model is based upon.

The thrust of this work was to build a tool to estimate the fragility of substation components in an explosive blast environment. Figure 8 shows a summary of the output from simulation code for horn gap switches in simulated substation as a result of a $1000 \mathrm{lb}$ Ammonium Nitrate Fuel-Oil (ANFO) bomb placed randomly about the perimeter of the substation. These switches normally sit about 40 feet above ground and have good line-of-sight from nearly all perimeter bomb locations. In these cases, where self-shielding from other substation components is minimal, the probability of failure for one of these switches is primarily a function of distance from the explosion. For components with good line-of-sight, Figure 8 suggests that generic fragility curves as a function of distance could be developed for components with unshielded line of sight. This is due to the minimal effects from the layout of the substation and its other components. Components located on the ground, such as the main and secondary transformers, exhibit a much greater dependence on local geometry and shielding effects from other components.
In summary, a software simulation tool was built that allows a skilled analyst to construct a simulated bomb by specifying its size, type, and fragment mass. Fragment speeds from the bomb were calculated using the CONWEP[1] blast simulation equations, and the directions of the fragments were drawn using a Monte Carlo technique. These fragments were traced through a simulated electric substation constructive solid geometry model using a ray-trace technique. Damage to components was calculated and tabulated. The final result for a given simulation is a table providing a summary of component hit and penetrated by bomb fragments. From this, the probability of failure for each component in the substation can be calculated as the number of failures divided by the total number of simulations. This simulation model can be used as a stand-alone vulnerability assessment tool for estimating damage in parametric studies that vary bomb makeup, size, and placement. It can also be used to evaluate shielding strategies. This work will also tie directly into the CIPRsim simulation environment which provides a 
comprehensive simulation model to predict the behavior of intertwined infrastructure systems. This gives INL a unique capability to simulate the Nation's infrastructures during terrorist activities and natural disasters.

\section{Copyright Statement}

This manuscript has been authored by Battelle Energy Alliance, LLC under Contract No. DE-AC07-05ID14517 with the U.S. Department of Energy. The United States Government retains and the publisher, by accepting the article for publication, acknowledges that the United States Government retains a nonexclusive, paid-up, irrevocable, world-wide license to publish or reproduce the published form of this manuscript, or allow others to do so, for United States Government purposes.

\section{REFERENCES}

[1] Technical Manual - Design and Analysis of Hardened Structures to Conventional Weapons Effects, The Departments of the Army, Air Force, and Navy, and the Defense Special Weapons Agency, Army TM 5-855-1, Air Force AFPAM 32-1147(I), Navy NAVFAC P-1080, DSWA DAHSCWEMAN-97, August 1998.

[2] Lee A. Butler, BRL-CAD Tutorial Series: Volume III - Principles of Effective Modeling, ARL-SR-119, Army Research Laboratory, September 2003.

[3] Image reproduced from Wikimedia Commons under the terms of the GNU Free Documentation License, Version 1.2, November 2002.

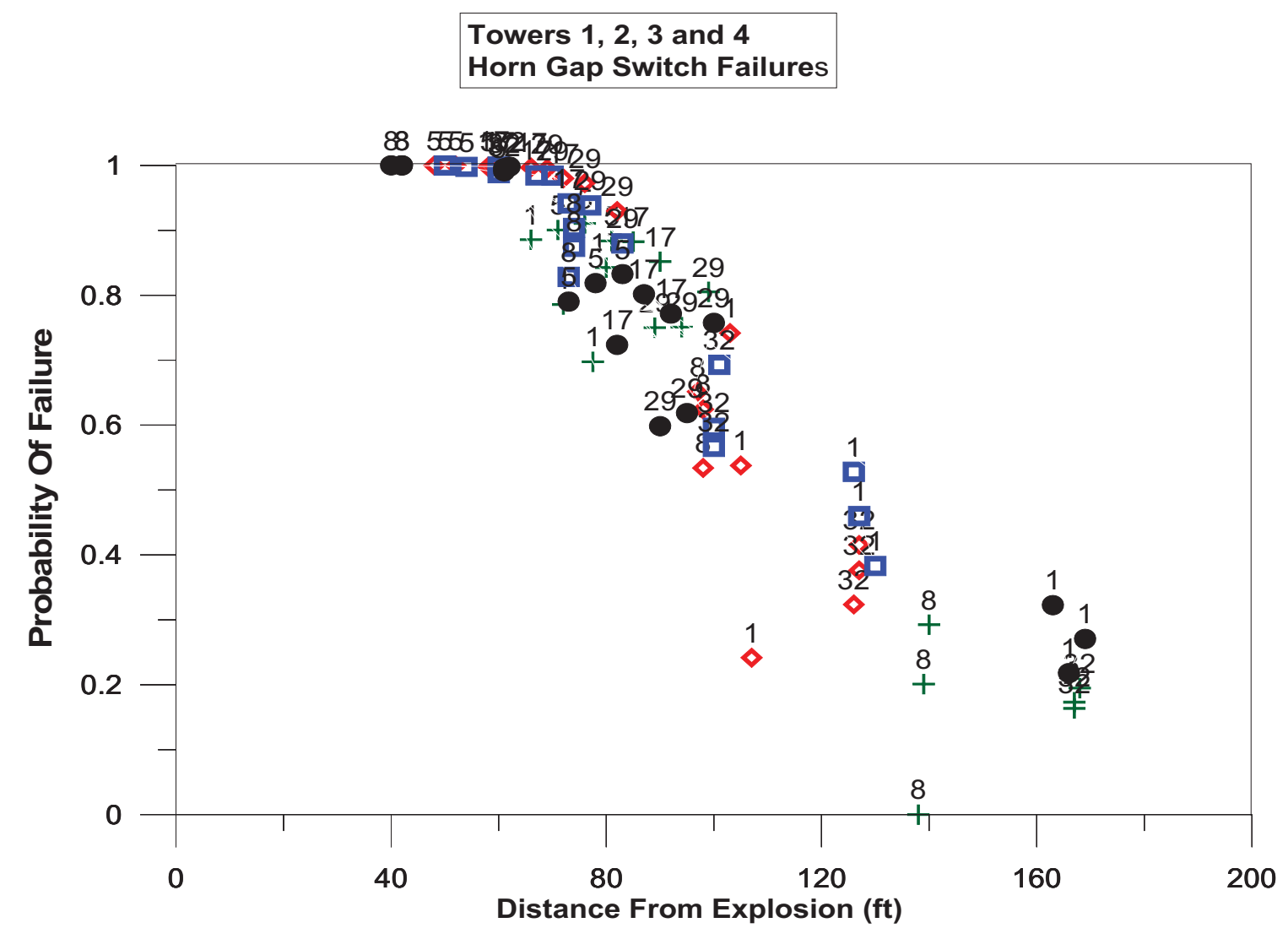

Figure 8 - Example failure distribution for horn gap switches for multiple bomb locations. 